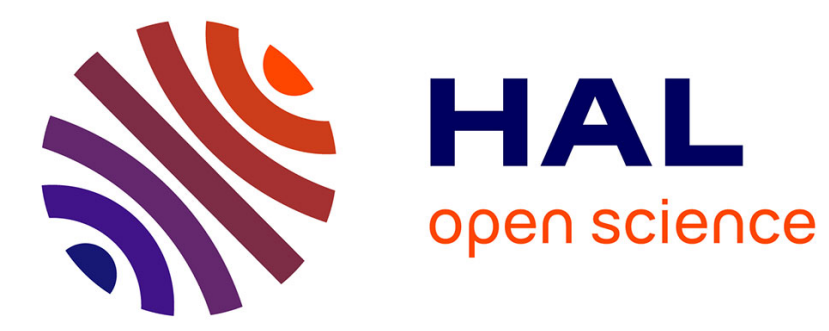

\title{
Donner un sens plus pur aux mots de la tribu Jean-Pierre Cuq
}

\section{To cite this version:}

Jean-Pierre Cuq. Donner un sens plus pur aux mots de la tribu. Dotoli giovani. La lisibilité du dictionnaire, Hermann, pp.51, 2015, 978-2-807-30715-5. hal-02119780

\section{HAL Id: hal-02119780 \\ https://hal-amu.archives-ouvertes.fr/hal-02119780}

Submitted on 4 May 2019

HAL is a multi-disciplinary open access archive for the deposit and dissemination of scientific research documents, whether they are published or not. The documents may come from teaching and research institutions in France or abroad, or from public or private research centers.
L'archive ouverte pluridisciplinaire HAL, est destinée au dépôt et à la diffusion de documents scientifiques de niveau recherche, publiés ou non, émanant des établissements d'enseignement et de recherche français ou étrangers, des laboratoires publics ou privés. 


\section{Jean-Pierre Cuq}

\section{Université Nice Sophia Antipolis}

Laboratoire I3DL EA 6308

\section{" Donner un sens plus pur aux mots de la tribu »}

\section{Introduction}

On me pardonnera de détourner ce vers célèbre de Mallarmé (Le tombeau d'Edgard Poe) pour résumer l'ambition que devrait avoir tout dictionnaire à visée professionnelle, et qui fut en tout cas celle des auteurs du Dictionnaire du français langue étrangère et seconde, que j'ai dirigé et qui est paru chez Clé international en 2003.

Je dirai dans un premier temps en quoi le DFLES fut à la fois l'émanation d'une tribu (de professionnels parlant et écrivant le même dialecte) et partie structurante vive de la tribu elle-même. Il s'agissait en effet de proposer à chacun des acteurs du champ du FLES un trousseau de clés de lecture actualisé, à la fois consensuel et engagé, de la littérature circulante.

En ce sens l'impératif d'écriture, qu'accrut la difficulté d'une écriture collective, fut de se rendre lisible au plus grand nombre possible de membres de la tribu, quelle que fût la position de chaque lecteur dans le champ.

Nous essaierons de montrer ensuite que la lisibilité d'un tel ouvrage, ne se travaille pas seulement au niveau de surface que représente l'harmonisation rédactionnelle, mais qu'elle est à l'origine même du projet d'écriture et se construit à chaque étape de l'élaboration du dictionnaire, que ce soit dans l'objectif (une lisibilité pour lire d'autres textes plus ou moins lisibles), dans le choix des rédacteurs (qui est légitime pour dire ou au moins proposer comment lire ?) ou dans le choix des items (quels sont les items nécessaires qui doivent être rendus lisibles et comment (nombre, taille, références,... ?).

1. Un ouvrage collectif

Le projet d'écriture de ce dictionnaire est né dans le séminaire de troisième cycle que j'animais à Aix-en-Provence à la fin des années 90. La question que je posais à mes étudiants était la suivante : pour vous aider dans la lecture des références de vos recherches et pour leur écriture, quels sont les termes que dont vous aimeriez disposer d'une définition fiable et actualisée ? Outre les relevés opérés dans leurs lectures Les étudiants ont alors dépouillé plusieurs dictionnaires ou glossaires en français ou en langue étrangère, au sein desquels ils ont conservé les items qui leur paraissaient les plus utiles : ils ont ainsi dressé une première liste d'environ 500 termes, sur laquelle un premier calibrage d'importance relative a été effectué. L'importance relative, sur une échelle à trois degrés (très important, moyennement important, peu important) ne visait pas l'évaluation du concept lui-même, mais celle de la nécessité de disposer pour lui d'une définition didactique convenable.

Dans un second temps, le questionnement a été associatif. Lorsqu'on m'a proposé en 2000 de prendre la présidence de l'association ASDIFLE, j'ai accepté à la condition que nous ferions quelque chose en commun, et, compte tenu de la préoccupation qui était alors la 
mienne, c'est le choix de la rédaction d'un dictionnaire qui a été retenu. Pour la plupart, les rédacteurs du DDFLES ont été choisis parce qu'ils étaient membres de l'ASDIFLE. Une association de recherche es en elle-même une sorte de microcosme, de tribu si on veut, qui se réunit autour d'un objet commun et qui doit disposer d'un langage partagé. Mais un certain nombre d'entre eux étaient des personnalités extérieures à l'association, qui ont été sollicitées en raison de leurs compétences reconnues dans leur domaine mais aussi pour marquer l'ouverture de l'association à l'intérieur de son champ disciplinaire et même à ses marges. Les rédacteurs de cet ouvrage appartenaient également à toutes les catégories de professionnels de la recherche et de l'enseignement du français langue étrangère et seconde, du professeur d'université en retraite jusqu'à l'étudiant de troisième cycle, en passant par les collègues de l'Alliance française, du réseau français à l'étranger, de l'inspection ou des IUFM. C'est ainsi non seulement le savoir de plusieurs générations de didacticiens, de chercheurs et de praticiens de tous horizons qui a été collecté et mis en forme, mais aussi celui des usagers directs des termes définis. Au total, plus d'une centaine de rédacteurs ont participé à la rédaction du DDFLES. A cette deuxième étape, une centaine d'items ont été rajoutés à la liste initiale.

Dans un troisième temps, un conseil scientifique a été constitué. II a travaillé par correspondance mais aussi lors d'un séminaire qui s'est tenu à Nancy en 2002. Le conseil a choisi de supprimer près d'une centaine des termes proposés aux étapes précédentes mais il en a rajouté un nombre à peu près équivalent. Ce qui fait que le DDFLES compte au total un peu plus de 600 items.

La composition du conseil scientifique ${ }^{1}$ tentait elle aussi de rendre compte assez largement du champ du FLE-FLS. Or, comme c'était déjà le cas pour le Dictionnaire de didactique des langues (Galisson et Coste, 1976) et bien qu'il existe certainement des lignes de partage intellectuel assez fortes, et sans aucun doute des filiations plus ou moins admises (ceux qui encore maintenant se sentent plus proches des pensées initiatrices de Galisson, de Porcher, de Rivenc, de Dabène ou de quelques autres),on ne peut pas dire qu'il y ait aujourd'hui plus qu'hier d'école(s) française(s) de didactique des langues au sens propre du terme, c'est-àdire rangée en plus ou moins bon ordre derrière un maitre à penser. De plus, la grande diversité des rédacteurs du DDFLES ne pouvait à l'évidence pas conduire à des choix d'école. Conformément à ce que je souhaitais, ce dictionnaire n'est donc pas le produit d'un courant unique de pensée didactique. II reflète au contraire une image polymorphe de la didactique du FLES et, plus largement de la didactique des langues telle qu'elle se présentait il y a une dizaine d'années en France. Cependant, on peut dire avec le recul que, sans que ce soit véritablement exprimé ou même voulu à l'origine, la rédaction d'un dictionnaire collectif correspondait en quelque sorte pour l'association, pour s'exprimer en termes bourdieusiens, à un désir de s'affirmer en tant qu'acteur majeur du champ, d'y prendre des positions scientifiques et donc de rendre lisible à d'autres acteurs l'objet didactique du FLES en des termes appartenant à son propre langage .

Le fait qu'il s'agît cependant d'une vision ouverte et plurielle a sans doute été une des raisons pour lesquelles, le DDFLES a été bien et largement accueilli, et qu'il n'a subi aucun de

\footnotetext{
${ }^{1}$ Marie-José Barbot (U. du Littoral Côte d’Opale), Robert Bouchard (U. Lumière Lyon 2), Francis Carton (U. Nancy 2), Jean-Pierre Cuq (U. De Provence Aix-Marseille 1), Pierre Dumont (U. Paul Valéry Montpellier 3), Elisabeth Guimbretière (U. Paris 7), Henry Holec (U. Nancy 2), Louis Porcher (U. Paris 3). Depuis, certaines universités ont pu changer de nom.
} 
ces ostracismes dont l'université est coutumière. En quelque sorte, sa diversité génétique, si je puis dire, lui assurait déjà une grande lisibilité. On peut dire qu'à travers ce dictionnaire, le groupe professionnel des acteurs du champ du FLE-FLS s'est parlé à lui-même, qu'il s'est dit comme tel à lui-même et aux autres.

Bien entendu, cette grande diversité de contributeurs a induit une certaine diversité stylistique, dont on pourrait sans doute relever des traces ici ou là. Mais le choix qui a été fait, et auquel je tenais particulièrement, de ne pas signer les articles, rendait nécessaire une certaine harmonisation de surface, que j'ai assurée. Toutefois, un bon nombre d'articles, surtout parmi les plus courts, sont de la plume d'un seul auteur, et n'ont pas eu à être retouchés. D'autres sont issus d'une réécriture de deux ou trois propositions. Dans tous les cas, et sous le contrôle du conseil scientifique qui a pu demander des modifications parfois relativement substantielles aux auteurs, j'ai respecté l'écriture et le fond de leurs propositions théoriques, même pour les articles sur lesquels il a pu arriver que je ne me sente pas totalement en accord.

\section{Un ouvrage d'affirmation disciplinaire}

Ce n'est un secret pour personne qu'au sein de l'université, la didactique du français langue étrangère et seconde (DFLES) souffrait (et souffre encore aujourd'hui mais dans une moindre mesure) d'un déficit de reconnaissance. Pas seulement au sein de l'université d'ailleurs : il suffit de lire, à chaque occasion, avec quel mépris la presse généraliste tient ce qu'elle appelle les "pédago ", coupables de tous les à peu près intellectuels, de tous les abandons et en premier lieu de celui du "savoir ", et responsables, bien entendu, des piètres résultats de l'école française dans les classements internationaux. Avec ses relents d'encre violette et de préaux humides, la pédagogie suscite sans doute une navrante et nostalgique bienveillance aux yeux de beaucoup, mais il ne faudrait tout de même pas, aux dires des vrais savants, qu'elle cache sous le terme ambitieux de didactique une prétention scientifique dont elle est à l'évidence indigne!

C'est également ce que pensaient (et pensent peut-être encore) la plupart des linguistes, pour ne rien dire des spécialistes de littérature, deux nobles piliers des universités de lettres françaises. Quant aux spécialistes de langues vivantes, il serait cruel de relever le pourcentage de thèses ou de travaux qui sont consacrés aux problèmes que pose leur transmission en regard de ceux qui sont consacrés à leur description ou à leur littérature.

Pourtant, c'est bien la conviction que le savoir accumulé sur la transmission des langues (leur appropriation par apprentissage et surtout, pour être conforme à l'étymologie du mot même de didactique, leur enseignement) contribue à une juste place à la connaissance des langues elles-mêmes qui justifie la réflexion en didactique des langues, et qui la place selon moi au même titre que la linguistique au sein des sciences du langage.

Pour ce qui concerne le français, une bonne part des concepts et des notions qui permettent de réfléchir cet enseignement et cet apprentissage à des non natifs sont des cas particuliers de l'enseignement et de l'apprentissage des autres langues vivantes. Mais I'histoire et l'expansion de cette langue d'une part, et le développement d'abord au sein d'organismes créés à cet effet (le BELC et le Crédif) en 1959, soit en pleine période de décolonisation, puis de filières particulières au sein des universités françaises à partir de 1983, a suscité l'éclosion 
de praticiens et de théoriciens de ce domaine particulier, qui se reconnaissent plus ou moins explicitement comme des acteurs de ce champ.

Or ce champ, comme tout champ linguistique, est structuré par des publications propres qui sont produites et lues par des professionnels qui occupent des positions différentes mais qui ont besoin de disposer d'un langage professionnel relativement stabilisé. Et on sait que c'est une gageüre en sciences humaines! Toutes les définitions qui ont été proposées dans le $D D F L E S$ ont donc été conçues avec le souci d'être intégrées et intégrables dans la réflexion plus large que constitue la didactique des langues mais d'être spécifiées autant que possible dans le champ du français langue étrangère. J'entends par là le souci des exemples (par exemple les articles unité capitalisable, variation) mais aussi et surtout celui de rendre lisible les publications du champ: des revues telles que Le français dans le monde, ou son supplément plus théorique Recherches et Applications, ELA (anciennement Etudes de linguistique appliquée) ou d'autres, d'aider les étudiants à la rédaction de leurs mémoires de recherche ou de leurs thèses.

On peut donc dire que la rédaction et la publication de ce dictionnaire ont correspondu à une période d'affirmation disciplinaire de français langue étrangère et seconde au sein d'un ensemble à prétention scientifique plus large, la didactique des langues. J'entends par discipline un découpage culturel et administratif tel qu'on peut le dire pour la discipline français dans le système d'enseignement primaire et secondaire français par exemple, mais avec les spécificités et les ambigüités que lui confère sa place initiale dans le système universitaire. Un peu paradoxalement, à l'heure où cette période volontariste semble malheureusement un peu s'essouffler, on observe aujourd'hui l'entrée officielle dans le système secondaire, sinon de la discipline en tant que telle mais au moins de la problématique dont elle est porteuse avec l'ouverture d'une option français langue étrangère et seconde au concours de recrutement des professeurs de français de l'enseignement secondaire (CAPES).

Je dirai pour conclure cette partie de mon propos qu'il me semble que de même qu'un dictionnaire de langue fournit à cette langue un appareillage qui la fortifie, le DDFLES a contribué à rendre visible et lisible le FLES en tant que discipline.

3. Le DDFLES, témoin de l'évolution conceptuelle de son époque

Pour s'affirmer légitimement en tant que discipline relativement autonome (au sens de domaine de réflexion scientifique cette fois), il est important de montrer qu'on dispose d'un appareillage conceptuel adéquat. Or, par sa nature même, la didactique du FLES est au croisement de plusieurs autres disciplines dont le caractère scientifique est plus ou moins affirmé : les langues vivantes, les sciences du langage, les sciences de l'éducation, la psychologie, la littérature. Il est donc naturel qu'un phénomène d'importation conceptuel ait toujours eu lieu entre ces domaines. Mais la question s'est historiquement débattue en ces termes: s'agit-il pour la didactique d'être une application d'une autre discipline, et notamment de la linguistique (la linguistique appliquée), ou s'agit-il de considérer ces disciplines comme des domaines de référence pour une réflexion didactique autonome? C'est la deuxième voie qui a fini par l'emporter en France, mais non sans combats acharnés. En d'autres termes, il s'agit : 
- d'admettre qu'on peut et parfois qu'on doit, utiliser des concepts et des notions issus d'autres disciplines mais en les réinterprétant si nécessaire en fonction du nouvel ensemble conceptuel auquel ils doivent s'intégrer. Par exemple, le concept de langue, qui est pour les linguistes un objet de description n'est jamais vu dans les dictionnaires de linguistique comme un objet d'enseignement et d'apprentissage, ce qu'il est fondamentalement pour les didacticiens.

- d'en créer en propre si nécessaire (exemple : immersion).

Comme toujours, c'est à la fois la forme et le fond qui assurent la compréhension

\subsection{La forme : le métalangage}

Nommer les concepts et les notions est une question importante car le métalangage choisi est un élément essentiel à leur lisibilité. On a constaté au fur et à mesure de la mise au point de la liste des items du DDFLES que le métalangage didactique n'est pas particulièrement ésotérique. Sans entrer dans les détails, on notera que comme en linguistique, ce métalangage a plusieurs origines :

- Le lexique courant, requalifié avec une acception particulière. (exemple, exercice, observation, immersion, légitimité, tâche, éclectisme...)

- Un lexique créé ad hoc qui peut alors provenir des disciplines de référence (orthoépie, renforcement, kinésique, mot, phrase...) ou être spécifique à la discipline. II répond alors bien entendu aux règles de formation du lexique français :

- en lexie simple : note, règle ...

- en composition : laboratoire de langue, faux débutant, tableau de feutre ...

- en dérivation : celle-ci peut être plus ou moins perceptible en synchronie et plus ou moins savante: apprenant, enseignant, guidance, vs interculturel, plurilinguisme, docimologie, noosphère...

- Les sigles : VGOS, DELF, TIC

- Les mots ou expressions d'origine étrangère : feed-back, portfolio, input, gestalt...

Dans ce cas, un équivalent français a systématiquement été proposé, qu'il soit couramment utilisé ou non dans la littérature spécialisée. A l'inverse, pour faciliter la lecture du dictionnaire par des membres étrangers de la communauté didactique du FLE, qui est, ne l'oublions pas, en majorité de langue première autre que le français des glossaires ont été proposés en fin de volume en allemand, anglais, espagnol, italien et portugais. Ces glossaires ont été écrits par des didacticiens parleurs natifs de ces langues. Pour l'italien et à titre d'exemple: Enrica Galazzi, Chiara Molinari, Christina Bosisio, toutes trois de I'Université Catholique de Milan, et Raffaele Spiezia, alors à I'université de Matera e Basilicata.

\subsection{Le fond : une importante évolution conceptuelle}

La rédaction du DDFLES a permis de rendre visible une forte évolution conceptuelle, notamment quand on le compare avec son beau et grand prédécesseur le Dictionnaire de didactique des langues que Robert Galisson et Daniel Coste avaient publié chez Hachette en 1976. Je reprendrai ici l'essentiel d'une étude de cette évolution que j'avais faite il y a quelques années. 


\subsubsection{La fin de la linguistique appliquée.}

Alors que le Dictionnaire de didactique des langues de Galisson et Coste comptait environ 625 items issus de la linguistique, soit près des deux tiers du dictionnaire, le DDFLES n'en propose qu'un peu plus de 150, soit moins du quart. Comme les deux ouvrages proposent environ 115 de ces items en commun, on peut voir que le DDFLES a supprimé un peu plus de 500 items relevant de la linguistique dans le même temps où il en proposait une petite cinquantaine de nouveaux. Entrent ainsi en lice deux parties récentes de la linguistique, la macrosyntaxe et l'analyse de la conversation, avec ses concepts afférents de face, d'intéraction et d'intéractivité.

Au début des années 2000 , le DDFLES rend ainsi compte du fait que la linguistique a nettement perdu de son influence dans la didactique des langues étrangères. Elle ne tient plus la place hégémonique qui était la sienne trente ans auparavant mais elle reste cependant la plus importante de toutes les disciplines de référence pour la didactique des langues, ce qui montre bien le cousinage des deux au sein des sciences du langage. Cependant les domaines linguistiques sollicités ont assez nettement évolué. Si les parties classiques de la linguistique (lexicologie, morphologie, phonétique, syntaxe) perdent une grande partie de leur importance quantitative, elles sont néanmoins encore présentes. Elles perdent aussi de leur importance relative par rapport à d'autres disciplines et en particulier la psycholinguistique. Compte tenu des orientations méthodologiques de la didactique contemporaine, on aurait pu s'attendre à ce que la sémantique et la pragmatique prennent une importance plus grande, mais il n'en est rien. La sociolinguistique elle-même ne fait pas de percée décisive. On peut dire enfin que l'apparition d'entrées nouvelles nous parait moins marquer un refus de la linguistique que la référence à une autre linguistique : celle de la parole plutôt que celle de la langue.

\subsubsection{Un glissement vers la psychologie cognitive}

Loin il est vrai derrière la linguistique, la psychologie est tout de même la seconde discipline de référence. Le $D D L$ présente presque une centaine de références, contre environ soixantedix seulement pour le DDFLES. Environ trente-cinq items sont communs aux deux. Le DDFLES conserve un certain nombre de concepts fondamentaux pour la discipline (ex; acquisition compréhension, mémoire...) mais montre un net renforcement du cognitivisme que marque l'entrée de plusieurs notions comme bas-haut, cognition, déclaratif, habileté, haut-bas, savoir, savoir déclaratif, savoir procédural, savoir-faire, surcharge cognitive). A noter aussi l'entrée de zone proximale de développement, appartenant au théoricien russe de la psychologie sociale, Vygotski, un auteur traduit en français seulement en 1985 et qui n'était donc que peu lisible chez nous auparavant.

\subsection{3. une relative percée des sciences de l'éducation.}

Les deux disciplines étant aussi très proches, et même souvent confondues dans les universités étrangères, il est parfois difficile d'attribuer les concepts à l'une ou à l'autre. Cependant le DDFLES est plus marqué par les sciences de l'éducation que le $D D L$ qui ne comprenait qu'une quinzaine d'entrées attribuées aux sciences de l'éducation. Sept de ces concepts sont en partage avec le DDFLES qui ajoute une vingtaine de concepts, généraux ou techniques comme éducation, formation, interdisciplinarité, motivation, non-directivité, remédiation, remue-méninges ou pédagogie différenciée. Le DDFLES n'a pas hésité non plus 
à introduire certains concepts très débattus, comme celui de transposition didactique, directement issu de la didactique des mathématiques, dont les écrits étaient peu lus à l'époque par les didacticiens du FLE.

\subsubsection{Un intérêt nouveau mais mesuré pour la sociologie}

Le $D D L$ ne comptait qu'un item relevant de cette discipline, l'éponyme sociologie qu'il partage avec le DDFLES. Celui-ci intègre treize items nouveaux, la plupart très inspirés de la sociologie de Bourdieu, introduite dans la didactique française par Louis Porcher dont on mesure ainsi l'influence: capital, classement, demande, espérance pratique, habitus, légitimité, marché, objectivation, objectiver, offre et produit. On note aussi l'entrée des concepts pourtant relativement anciens d'ethnocentrisme et de représentation.

\subsubsection{Des traces d'autres disciplines}

Outre quelques concepts généraux (information, praxéologie...) le DDFLES se fait l'écho de disciplines nouvelles ou nouvellement convoquées: l'ethnographie de la communication, (kinémique, kinésique, proxémique), les neurosciences et la psychanalyse.

Au total, le nombre de ces items n'est pourtant pas significatif de l'implication forte de nouvelles disciplines. Ont ainsi disparu les items du $D D L$ venant des mathématiques (et non pas de la didactique des mathématiques). On peut peut-être voir en cela un signe du triomphe en France d'une didactique qualitative au détriment d'une didactique plus quantitative.

\subsubsection{Une montée en puissance de la didactique}

Le basculement le plus net s'observe en didactique, qui n'hésite pas à tenter même de se renommer elle-même avec le concept galissonien de didactologie.

Là où le $D D L$ présente environ 180 entrées d'origine purement didactique, le DDFLES en offre 285 , c'est-à-dire environ quarante pour cent du total. Un peu plus de cent entrées sont communes aux deux dictionnaires et représentent sans doute une sorte de noyau dur disciplinaire, qui a été transmis d'une génération à l'autre.

\section{- Aspects généraux et méthodologiques}

Les deux dictionnaires ont bien entendu un grand nombre d'items didactiques communs, qui concernent essentiellement les fondements de la discipline et de la méthodologie de l'enseignement (actif, audio-oral, audio-visuel, bilinguisme, didactique, direct, exploitation, intensif, individualisation, méthode, méthodologie, moments, pédagogie, lecture, précoce, verbo-tonal...), l'apprenant ou l'enseignant (apprenant, apprentissage, autonomie, besoin, captif, débutant, faux débutant, stratégie...), les techniques (autoscopie, cours, exercice, explication, réponse, simulation, systématisation, technique, traduction, etc.).

Mais l'abandon de beaucoup d'items présents dans le DDL tels qu'enseignement programmé, filtré, lavé, ou micro-conversation, indique aussi une certaine évolution méthodologique.

On s'arrêtera surtout sur les nouveaux items du DDFLES, au nombre d'environ 150. Ils illustrent en partie la notion de centration sur l'apprenant (apprendre à apprendre, autoapprentissage, autocorrection, autonomisation, centration, élève nouvellement arrivé, etc.) 
et montrent aussi l'évolution du rôle et des relations des participants à la classe (acteur, activité, contrat, face-à-face) et surtout de l'enseignant (animateur, conduite de classe, conseil, guidage, tuteur).

Ils pointent aussi des façons d'enseigner ou des méthodologies non répertoriées ou faisant seulement l'objet de parties d'articles dans le DDL (apprentissage communautaire, apprentissage par la réaction physique totale, approche communicative, approche naturelle, approche relationnelle, approche systémique, éclectisme, éveil au langage, jeu de rôles, méthode par le mouvement, méthode silencieuse, méthodologies non conventionnelles, pédagogie des grands groupes, pédagogie en contexte, suggestopédie).

On note aussi des concepts généraux redéfinis et renommés (milieu, situation de communication, français sur objectifs spécifiques), des éléments relevant de la politique linguistique ou de la francophonie, ou de façons nouvelles d'organiser l'enseignement (classe bilingue, échange éducatif, formation ouverte et à distance, francité, francitude, francophonie, Francophonie, immersion, institution, langue voisine...

Enfin, apparaissent dans le DDFLES des éléments théoriques ou méthodologiques pas forcément nouveaux, mais ne disposant pas d'une entrée dans le DDL (activité, bain, but, heuristique, cursus, manuel, prérequis, réécriture, réemploi, repérage, scénario, stratégie d'enseignement, syllabus), ou marquant l'apparition de concepts nouveaux en didactique (curriculum, éthique, hétéroglotte, ingénierie, noosphère, référentiel, scénario, stratégie d'enseignement, syllabus).

\section{- Aspects culturels}

Ils nous semblent très symptomatiques de l'évolution de la discipline. On n'ignore pas que R. Galisson a lui-même insisté pour appeler la discipline didactologie des langues et des cultures, et il a sans doute fortement contribué à la montée de l'intérêt pour les aspects culturels qui apparait clairement dans le DDFLES. Pourtant en 1976, il n'y avait dans le DDL que quatre termes relevant de ce domaine (acculturation, civilisation, culture, enculturation), dont seul le dernier n'est pas dans le DDFLES. Ce dernier en revanche fait entrer treize items nouveaux (aculturation, altérité, connaissance partagée, croyances, décentration, déculturation, emblème, empathie, étranger, français, interculturel, savoirêtre, stéréotype).

\section{- Evaluation}

Cet aspect de la didactique voit aussi légèrement augmenter son quota de termes puisqu'ils passent de quinze à vingt. Outre les anciens auto-évaluation, capitalisable, docimologie, erreur, évaluation, examen, faute, test, unité capitalisable, validation, les items entrés dans le DDFLES concernent comme il est normal des certifications, des référentiels ou des tests qui n'existaient pas à l'époque du $D D L$ (cadre européen de référence, DELF-DALF, portfolio européen des langues, $T C F, T E F$ ) et témoignent aussi de l'intérêt pour la réflexion évaluative avec barème, certification, diagnostic, échelle scolaire, évaluateur, notation, note, pronostic.

\section{- Aspects technologiques}

Assez curieusement, les items consacrés aux aspects technologiques sont en régression (dix contre vingt-cinq). En plus de six entrées communes aux deux dictionnaires (figurine, film, 
laboratoire, multimédia, tableau de feutre, technologie), les entrées originales du DDFLES concernent la nouvelle dénomination générale de l'aspect technologique de la didactique (TIC-TICE), une de ses techniques majeures (vidéo) et un concept afférent (dispositif). Elles notent aussi un terme francophone qui s'est imposé (didacticiel) mais qui n'existait pas à l'époque du DDL, et enfin le concept récent de centre de ressources.

Ont en revanche disparu des technologies ou des dénominations aujourd'hui obsolètes (circuit, diascope, hardware, machine à enseigner, passe-film, software, Suvag-Lingua), ou des matériels dont l'usage est devenu si courant qu'il n'apparaissait pas utile aux auteurs du DDFLES de les définir. Signe de la rapidité de l'évolution technologique, certains sont euxmêmes en voie d'archéologisation depuis la sortie du DDFLES: (cassette, magnétoscope, rétro-projecteur, tableau magnétique, vidéo-cassette).

\section{CONCLUSION}

Grâce à cet énorme effort de conceptualisation accompli depuis près d'un demi-siècle maintenant, la didactique des langues, et en son sein plus particulièrement la didactique du français langue étrangère et seconde s'est peu à peu fait une place dans le champ de la réflexion en sciences humaines. La publication d'un dictionnaire spécifique au français langue étrangère et seconde, outillage majeur d'un champ linguistique ou intellectuel, a incontestablement aidé cette discipline à rendre plus lisible sa littérature interne mais l'a aussi aidée à se rendre plus lisible auprès des chercheurs d'autres disciplines. En donnant un sens plus " pur » à ses mots, elle a aussi façonné la tribu elle-même des didacticiens du FLES. Mais en sciences humaines comme et peut-être plus vite qu'ailleurs, le sens des concepts et des notions évolue, et il est sans doute illusoire de leur donner et plus encore de leur conserver un sens " pur ". C'est sans doute alors dans l'évolution sémantique elle-même, en ce qu'elle dégage progressivement le sens des obsolescences, qu'il faut chercher une valeur à cette image. La lisibilité d'un dictionnaire paru en 2003 est-elle encore suffisante pour éclairer la lecture de textes plus jeunes de dix ans ? Rien n'est moins sûr mais il appartiendra à une autre génération de didacticiens de le montrer. Sans cet effort, ils pourraient bien se rendre assez vite illisibles...

\section{BIBLIOGRAPHIE}

Cuq, J.-P (dir.) : Dictionnaire de didactique du français langue étrangère et seconde, CLE International, 2003.

Cuq, J.-P. : « trente ans d'évolution de la didactique des langues étrangères et secondes en France vues à travers deux dictionnaires ", dans Bouffard P. et Knoerre H., La Revue de I'AQEFLS, volume 25, n², Montréal, 2005, pp. 45-61.

Galisson R. et Coste D. : Dictionnaire de didactique des langues, Hachette, F, 1976. 